\title{
Induction of Sirt1 and PGC1 $\alpha$ Signalling Pathway in Cardiac Mitochondrial Biogenesis by Aspirin
}

\author{
Pratibha Kamble ${ }^{1 *}$ and Sameer Kulkarni² \\ ${ }^{1}$ Division of Cardiac Surgery, Ohio State University, Columbus, USA \\ ${ }^{2}$ Department of Biochemistry, Grant Medical College \& Sir J.J. Group of Hospitals, India
}

${ }^{*}$ Corresponding author: Pratibha Kamble, Division of Cardiac Surgery, Ohio State University, Columbus, USA, Tel: +1 614-721-3648; E-mail: kamblepratibha11@gmail.com

Received date: January 17, 2018; Accepted date: February 02, 2018; Published date: February 12, 2018

Copyright: @ 2018 Kamble P, et al. This is an open-access article distributed under the terms of the Creative Commons Attribution License, which permits unrestricted use, distribution, and reproduction in any medium, provided the original author and source are credited.

Citation: Kamble P, Kulkarni S (2018) Induction of Sirt1 and PGC1 $\alpha$ Signalling Pathway in Cardiac Mitochondrial Biogenesis by Aspirin. Eur Exp Biol Vol. 8 No. 1:6

\section{Abstract}

Aims: We hypothesized that acetyl salicylic acid (Aspirin, ASA) treament has ability to induce Sirtuin 1, 4 (Sirt1 and Sirt4) and it downstream targets, peroxisome proliferatoractivated receptor-gamma co-activator-1 $\alpha \quad$ (PGC1 $\alpha)$ and mitochondrial transcription factor A (Tfam) gene in cultured HL1 cardiomyocytes. We also assume that hydrogen peroxide $\left(\mathrm{H}_{2} \mathrm{O}_{2}\right)$ formed during redox reactions is inducer of Sirt1 gene.

Methods and Results: Atrial Cardiomyocyts HL1 cells were cultured in Claycomb medium with $10 \%$ FBS, $100 \mu \mathrm{mol} / \mathrm{L}$ norepinephrine, and $4 \mathrm{mmol} / \mathrm{L}$ L-glutamine (Invitrogen, Carlsbad, CA) in gelatin coated flasks. Cells were maintained at $37^{\circ} \mathrm{C}$ in an atmosphere containing $5 \% \mathrm{CO}_{2}$. Cells were then incubated with either $50 \mu \mathrm{M}$ and $0.25 \mathrm{mM}$ ASA for 48 $h$ in the same medium. Respective controls were maintained with alcohol alone. At the end of the treatment, the medium was removed and the cells were washed with PBS and harvested in Trizol ${ }^{\circledR}$ for isolation of RNA. RT-PCR was performed for the analysis of gene expression. Our results in cultured HL1 cardiomyocytes showed ASA treament induced Sirt1 and Sirt4 genes via $\mathrm{H}_{2} \mathrm{O}_{2}$ generation. Increase in Sirt1 gene activated PGC1 $\alpha$ and Tfam gene. ASA have also induced antioxidant enzymes, glutathione peroxidase (GPX) and catalase (CAT) gene.

Conclusion: We conclude from our results that ASA is a potential anti-atherosclerotic drug and an increase in Sirt1 gene further suggest that it can induce anti-inflammatory action. It can also interfere with $\mathrm{Nfkb}$ signaling pathway that can prevent foam cell formation. However, specific overexpression of PGC1 $\alpha$ and Tfam gene in HL1 cardiomyocyte can increase mitochondrial biogenesis and prevent the development of heart failure.

Keywords: Hydrogen peroxide; Acetyl salicylic acid; Sirtuins; PGC1 $\alpha$; Mitochondrial biogenesis

\section{Introduction}

Cardiovascular disease has been assosiated with blood lipids disorder [1,2]. Previous reports suggests that caloric restriction can decrease the mortality rate from coronary artery disease (CAD) which is evidenced by change in serum Tchol, low density lipoprotein cholesterol (LDL-C), high density lipoprotein cholesterol (HDL-C), triglyceride (TG) and blood pressure (BP) parameters that usually elevates with age [3].

Cardiomyopathy attends from changes in calcium signaling to inflammation to cardiomyocyte toxicity. Several studies also links this abberent change to mitochondrial pathogenesis, which inhibits the expression of nuclear and mitochondrial encoded genes involved in mitochondrial biogénesis [4]. Heart is a organ of high energy demand that acts through transactivation of genes, which includes nuclear respiratory factor (NRF)-1 and (NRF)-2, more than that PGC1 $\alpha$ and Tfam plays critical role in mitochondrial biogénesis [4-6]. There are several such reports that documents various pharmacological drugs includes statins, NSAIDS and a natural component of red wine resverastrol which can value in potential anti-atherosclerotic therapy [1]

ASA is analgesic, antipyretic and antithrombotic drug which is traditionally used for the treatment of artherosclerotic diseases [7]. It is ethyl ester that is widely applicable from 150 years to prevent inflammation. The mechanism of action of ASA is that it reacts with cycloxygenase and prevents inflammation by acetylating the active site of the enzyme and reduces the formation of thromboxanes and prostacyclins. The antiplatelet function, along with endothelial activation, cell proliferation and leucocyte migration, contributes to its clinical manifestation $[7,8]$. Liu et al. reported that fractalkine is responsible for severity of atherosclerosis and ASA treatment reduces the fractalkine expression in the aorta.

ASA is enzymatically hydrolyzed by paraoxonase 1 (PON1) to Salicylic acid (SA). ASA has life span of about 15 mins and SA has half life of several hours and also has major benefits as a hydroxyl radical trapper. However, it is known to be metabolized by liver microsomes enzymatically to 2,3- and 2,5-DHBA by hydroxyl radicals. These dihydroxybenzene derivatives, such as 
(2,3- or 2,5-DHBA) are expected to autooxidize to form quinones and reactive oxygen species (ROS) [9].

In our previous studies we have documented that cultured liver cells treated with ASA resulted an increase in Sirt1, PGC1 $\alpha$ and Nqo1 gene through PON1 and aryl hydrocarbon receptor (AhR) dependent manner. However, PON1 and AhR siRNA transfection inhibited the expression level of these genes. Thus, studies confirmed our hypothesis that ASA and SA produced $\mathrm{H}_{2} \mathrm{O}_{2}$, which was inducer of Sirt1. We have also reported that increase in these transcription factors can contribute in mitochondriogenesis. Furthermore, our studies on Mapkinase and $\mathrm{NfkB}$ signaling pathway also showed that ASA increases Mapkinase pathway and inhibits NFkB [10].

Based on our previous findings our current attempt is to study the ability of ASA in cardiac cells. In that matter we have studied that the drug ASA could induce Sirt1, and its downstream targets, PGC1 $\alpha$ and Tfam which are essential mitochondrial genes. Moreover, Sirt4 which is a protein of mitochondrial origin and antioxidants such as CAT and GPX are also studied.

\section{Materials and Methods}

\section{Reagents}

ASA and all other chemicals were purchased from SigmaAldrich Chemical Co. (St Louis, MO). HL1 cells were purchased from the American Type Culture Collection (ATCC, Manassas, VA). All other reagents used were of analytical grade. PCR primers and cell culture reagents were obtained from Invitrogen (Carlsbad, CA).

\section{Cell culture and treatment}

Atrial Cardiomyocyts HL1 cells were cultured in Claycomb medium with $10 \%$ FBS, $100 \mu \mathrm{mol} / \mathrm{L}$ norepinephrine, and 4 $\mathrm{mmol} / \mathrm{L}$ L-glutamine (Invitrogen, Carlsbad, CA) in gelatin coated flasks. Cells were maintained at $37^{\circ} \mathrm{C}$ in an atmosphere containing $5 \% \mathrm{CO}_{2}$.

\section{Incubation HL1 cardiomyocyte}

HL1 cardiomyocytes were incubated with either $50 \mu \mathrm{M}$ and $0.25 \mathrm{mM}$ ASA for $48 \mathrm{~h}$ in the same medium. ASA was dissolved in absolute alcohol. Respective controls were maintained with alcohol alone. At the end of the treatment, the medium was removed and the cells were washed with PBS and harvested in Trizol ${ }^{\circledR}$ (Invitrogen, Carlsbad, CA) for RNA isolation.

\section{Sirt4, PGC1 $\alpha$, Sirt1, Tfam, AhR, CAT and GPX gene expression}

Total RNA was extracted from cells after ASA treatment by using Trizol (Invitrogen, Carlsbad, CA) according to the manufacturer's protocol. First strand CDNA synthesis was performed from $1 \mu \mathrm{g}$ total RNA. Reverse transcriptase products were subjected to PCR amplification with Ready Mix PCR Master Mix (Invitrogen, Carlsbad, CA). Oligonucleotide primers were designed on the basis of the cDNA sequences reported in the Gene Bank database. Primers for Sirt4, PGC1 $\alpha$, Sirt1, Tfam, AhR, CAT and GPX were designed by using Invitrogen Oligo Perfect ${ }^{\circledR}$. RT-PCR was performed for the analysis of gene expression (Table 1).

\section{Statistical analysis}

All experiments were performed atleast 3 separate times with triplicate measurements. The data are expressed as mean \pm S.D. and student's t test was applied for significance at $\mathrm{P}<0.05$.

Table 1. List of oligonucleotide primers used for RT-PCR.

\begin{tabular}{|c|c|c|c|}
\hline Species & Target & Forward Primer & Reverse Primer \\
\hline \multirow[t]{7}{*}{ Human } & Sirt 4 & 5'-TCCCCAGACACCCTGTTTTA-3' & 5'-GCAATTTCCCAAGTCTCTG-3' \\
\hline & PGC1a & 5'-GCAGCAGTTCCATGAAGACA-3' & 5'-TAGGGTGGTTTGTGGTTGGT-3' \\
\hline & Tfam & 5'-GGGTTCCAGTTGTGATTGCT-3' & 5'-TGGACAACTTGCCAAGACAG-3' \\
\hline & Sirt 1 & 5'-TGGAACAGGTTGCGGGAATC-3' & 5'-TCAGCTGGGCACCTAGGACA-3' \\
\hline & GPX & 5'AGGCGAGTTTCCCCACTAAT-3' & 5'- ACACGGTGAAACCCTGTCTC-3' \\
\hline & CAT & 5'-AGGCCAGTCCTGACAAAATG-3' & 5'-GAATCTCCGCACTTCTCCAG-3' \\
\hline & GAPDH & 5'-ACCCAGAAGACTGTGGATGG-3' & 5'-CACATTGGGGGTAGGAACAC-3' \\
\hline
\end{tabular}

\section{Results}

\section{ASA induces genes involved in the regulation of mitochondrial function}

Sirt4, PGC1 $\alpha$, Sirt1, Tfam and AhR gene expression in HL1 cells: Sirt4 is a mitochondrial protein that utilizes $\mathrm{NAD}^{+}$for carrying out ADP-ribosylation of glutamate dehydrogenase (GDH) in mitocondria [11,12]. Treatment of HL1 cells with $50 \mu \mathrm{M}$ ASA for $48 \mathrm{~h}$ resulted in significant increase in Sirt4 $(5.11 \pm 0.2$ fold) as compared to control counterpart ( $1 \pm 0.07)$ (Figure 1A).

PGC1 $\alpha$ are expressed abundantly in heart $[13,14]$ and it powerfully stimulates mitochondrial gene expression and biogenesis. Significant increase in PGC1 $\alpha$ gene expression was 
observed at $50 \mu \mathrm{M}(2.863 \pm 0.1)$ however, $0.25 \mathrm{mM}(0.8 \pm 0.1)$

ASA treatment for $48 \mathrm{~h}$ showed decrease as compared to control (1 \pm 0.1 ) (Figure 1B).
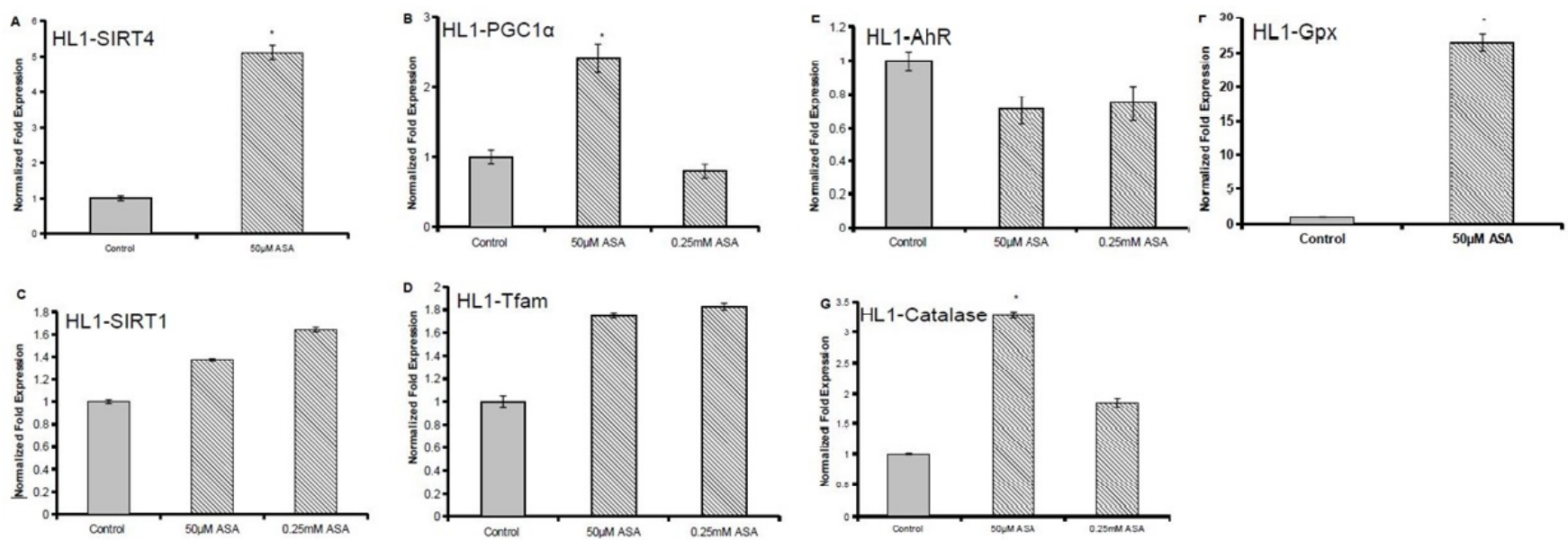

Figure 1(A-E). Induction of Sirt4 \& Sirt1 and its downstream targets PGC1 $\alpha$, Tfam in HL1 cardiomyocyte. Increase in antioxidant enzymes, GPX and CAT. The experimental set up included treatment of HL1 cardiomyocyte with different concentrations of ASA ( $50 \mu \mathrm{M}-0.25 \mathrm{mM}$ ) for $48 \mathrm{~h}$. Sirt4 and PGC1 $\alpha$ were induced in HL1 cardiomyocyte (A \& B respectively). Sirt1 and Tfam genes were induced in HL1 cardiomyocyte ( $C \& D$ respectively). AhR gene expression did not show any increase in gene expression in HL1 cardiomyocyte (E). Induction of GPX and CAT gene in HL1 cardiomyocyte treated with $50 \mu \mathrm{M}-0.25 \mathrm{mM}$ ASA (F \& G respectively). Values are expressed as mean \pm SD $(n=3)$. Statistically significant changes between untreated and treated sets are marked as $*(P<0.05)$.

Sirt1, a member of the conserved sirtuin family of $\mathrm{NAD}^{+}$ dependent histone deacetylases that deacetylates other proteins, including PGC1 $\alpha$ responsible for oxidative metabolism in heart [15]. Sirt1 gene showed an significant increase in both $50 \mu \mathrm{M}(1.37 \pm 0.01)$ and $0.25 \mathrm{mM}$ ASA $(1.64 \pm 0.02)$ compared to control counterpart $(1 \pm 0.02)$ (Figure 1 C).

Tfam is a mitochondrial transcription factor A protein that increases the number of mitochondria and can prevent heart disease4. Significant increase in Tfam gene expression was observed in $50 \mu \mathrm{M}(1.75 \pm 0.02$ fold) and $0.25 \mathrm{mM}$ ASA (1.83 \pm 0.03 fold) treated samples as compared to control $(1 \pm 0.05)$ (Figure 1D). However, AhR gene was decreased at both $50 \mu \mathrm{M}$ $(0.71 \pm 0.08)$ and $0.25 \mathrm{mM}$ ASA $(0.75 \pm 0.1)$ as compared to control countepart $(1 \pm 0.05)$ (Figure 1E).

\section{ASA activates enzymatic antioxidant program}

GPX and CAT gene expression in HL1 cells: HL1 cells showed increase in GPX expression $(26.41 \pm 1.1)$ in $50 \mu \mathrm{M}$ ASA as compared to control $(1 \pm 0.01)$ (Figure 1F). CAT is antioxidant enzyme that is located in cellular peroxisomes, which catalyzes the dismutation of $\mathrm{H}_{2} \mathrm{O}_{2}$, forming $\mathrm{O}_{2}$ and $\mathrm{H}_{2} \mathrm{O}$ [21]. HL1 cells treated with $50 \mu \mathrm{M}(3.28 \pm 0.05)$ and $0.25 \mathrm{mM}$ ASA (1.84 \pm 0.07$)$ showed significant increase in CAT gene expression as compared to control (1 \pm 0.01 ) (Figure 1G).

\section{Discussion}

Previous studies by Grube et al. have reported that there are organic cation transporter novel type 2 (OCTN2) protein expressed in human heart which contribute in cardiac uptake of cardiovascular drugs. However, the uptake of drugs by OCTN2 is poorly understood.

In this study we demonstarte that ASA metabolic conversion is not AhR dependent process in HL1 cardiomyocytes. Since AhR serves as a dominant receptor for various aromatic compounds including drugs however, in this particular study were AhR gene expression is decreased signifies that in HL1 cells this receptor via cyp1a1 might not be involved in metabolism of ASA to SA and 2,3 DHBA. Therefore, other drug transporting protein might be responsible for uptake which is then further by redox mechanism forms $\mathrm{O}_{2}^{-}$and $\mathrm{H}_{2} \mathrm{O}_{2}$. The $\mathrm{H}_{2} \mathrm{O}_{2}$ generated might be responsible for inducing Sirt1 and Sirt4 gene. Additionally, there are several reports which suggest that $\mathrm{H}_{2} \mathrm{O}_{2}$ can act as a activator of HDACetylases that includes Sirtuins 1 \& 4 in cardiac myocytes [16-18].

The concept that ROS have "purposeful" roles as "regulators" of cell function or as "signalling molecules" has gained significant recognition over past several years from studies done in laboratories worldwide [19].

Zhang et al. founded that oxLDL and $\mathrm{H}_{2} \mathrm{O}_{2}$ treatment increased the Sirt1 level in HUVECs. Meilhac et al. have reported in rabbit femoral arterial smooth muscle cells (RFASMC), CAT protein, $m R N A$, and the enzyme activity were increased in response to oxidized linolic acid (13-hydroperoxy-9,11octadecadienoic acid [13-HPODE] and 13-hydrooxy-9,11octadecadienoic acid [13-HODE], MO-LDL, or $\mathrm{H}_{2} \mathrm{O}_{2}$.

Thrombin and ET1 are potent vascular smooth muscle cell mitogens whose signalling is dependent on G-protein coupled receptors [20-22]. Thrombin generates ROS in both endothelial 
cells [19] and smooth muscle cells. Patterson et al. demonstarted the ability of thrombin to stimulate $\mathrm{O}_{2}^{-}$and $\mathrm{H}_{2} \mathrm{O}_{2}$ production in vascular smooth muscle cells that is associated with the $\mathrm{NAD}(\mathrm{P}) \mathrm{H}$ oxidase activity required for thrombininduced mitogenesis. ET1 also known to increase intracellular ROS production in cardiac myocytes by a p21RAS- dependent mechanism. There study showed that ET1-induced ROS is essential for the induction of c-FOS expression. In another study they found that ROS appear to mediate the inotropic effects of ET1 on the myocardium [19]. There are reports which suggests that acetylcholine activates ATP-sensitive $\mathrm{K}^{+}$channels and increase mitochondrial ROS that might function as an intracellular signal for acetylcholine-induced preconditioning in cardiomyocytes [23,24]. Exogeneous $\mathrm{H}_{2} \mathrm{O}_{2}$ (usually in the millimolar range) has been shown to induce tyrosine phosphorylation and activation of the PDGF- $\alpha$, PDGF- $\beta$, EGF receptors [19].

Thus moderate induction of Sirt1 gene expression in our studies suggests that the protein can control angiogenic function. This profile can also maintain normal endothelial function and can be vasoprotective [25].

Literature also suggests that cardiac specific overexpression of Sirt1 can prevent age dependent increase in cardiac hypertrophy, apoptosis and cardiac dysfunction however higher levels of Sirt1 induce cardiomyopathy [26].

Moreover, ASA at given dose level also increased PGC1 $\alpha$ gene expression. It suggets that Sirt1 decreases PGC1 $\alpha$ acetylation, as well as increases PGC1 $\alpha$ activity and induces other genes that are responsible for oxidative phosphorylation, energy homeostasis and mitochondrial biogenesis in cardiac muscle $[26,27]$, followed by an increase in expression of GPX, CAT and enzymes responsible for GSH synthesis $[27,28]$. Thus an increase in GPX and CAT gene expression in HL1 cells in our studies is suggestive of increased PGC1 $\alpha$ induced effect. Further it can be said that increase in Sirt1, PGC1 $\alpha$ and Tfam gene might regulate energetic pathway and we also assume that low levels of ROS formed by ASA treatment can play an important role in energetic recovery which is also required during myocardial ischemia.

One such group by Klawitter et al. have also explained importance of ROS signalling to enhance bioenergetic recovery as it increases the efficiancy of contraction. In thier studies they have reported that antioxidants such as, 1,2 dihydroxybenzene-3,5-disulfonate (Tiron), or N-acetyl-L-cysteine given before ischemia, reduced ATP recovery which was followed by decrease in contraction efficiency compared to controls.

Interestingly, there are reports which suggest that PGC1 $\alpha$ null mice develops early symptoms of heart failure, especially activation of fetal program of cardiac gene expression and highest level of circulating atrial natriuretic peptide (ANP) a marker of cardiac dysfunction [29,30]. Reports also suggest that loss of PGC1 $\alpha$ in knockout mice leads to defects in cardiac mitochondria and energetics, which inturn causes heart failure. The effects on oxidative and mitochondrial variables are mediated by Nrf1 and 2 and estrogen -related receptors (ERR) $\alpha$ [31].
Furthermore, Tfam which is mitochondrial transcription factor $A$ an increase in this gene supports the notion that both genes can regulate above process and can increase the number of mitochondria during cardiac condition and improve heart development. Tfam can also bring about replication and transcription of mitochondrial DNA [4].

PGC1 $\alpha$ is also implicated in heart function and pathogenesis of cardiac failure. During chronic pressure overload such as in chronic hypertension, PGC1 $\alpha$ gene controls fatty acid oxidation and Tfam gene increases number of mitochondria are downregulated which is consistant with the fact that mitochondrial respiratory function is reduced during end-stage heart failure [32].

Li et al. have reported that increased Sirt1 protein expression was accompanied by severe cardiac hypertrophy in older spontaneous hypertensive rat (SHR). To this possibility they further said that hyperexpression of Sirt1 in the heart of SHR may be a compensatory reaction in hypertension-induced cardiac hypertrophy.

Jaichander et al. have reported that ASA induces AhR serves as inducer of PON1 and Apoa1 in HepG2 cells. We have also hypothesized in our previous studies in liver cells the mechanism of action of ASA. Our studies depicted $\mathrm{H}_{2} \mathrm{O}_{2}$ induced Sirt1 and PGC1 $\alpha$ in HepG2 cells and primary hepatocytes.

We assume from present studies that as primary hepatocytes are highly permeable to PON1 and its other forms PON2 and PON3 the enzyme might be involved in metabolizing ASA to SA and DHBA. DHBA might be further transported to cardiac cells by drug transporters which by spontaneous redox reaction generated $\mathrm{O}_{2}^{-}$and $\mathrm{H}_{2} \mathrm{O}_{2}$. The $\mathrm{H}_{2} \mathrm{O}_{2}$ formed might be involved in inducing all the mitochondrial regulatory protein such as Sirt1, PGC1 $\alpha$, Tfam and Sirt4 which are usually highly expressed in heart.

ROS is also involved in PGC1 $\alpha$ expression, and this gene is known to limit the accumulation of ROS and prevents oxidative damage, via induction of other genes that limits ROS production and increasing ROS scavenging. Additionally forced decrease of adenosine-5' triphosphate (ATP) can also induce PGC1 $\alpha 31$ thus it can be speculated that an increase in PGC1 $\alpha$ gene after ASA treatment can contribute in replinising energetic demand of failing heart.

Nitric oxide is also a potant bioactive gas, which is known to be protective during ischemic conditions. It has been reported that nitric oxide can induce PGC1 $\alpha$ gene and mitochondrial biogenesis in fibroblasts and adipocytes which also suggest that it could happen in other tissues [33,34].

Thus increase in these transcriptional protein mainly Sirt1 can improve cardiac function through effects on multiple pathways that includes improved vasorelaxation (possibly through $\mathrm{K}^{-}$ channel inhibition), anti-inflammatory activity on macrophages and foam-cell formation. As Sirt1 regulates PGC1 $\alpha$, a central factor in controlling energy state and contractile function in cardiac muscle [26] an increase of these genes in our studies could be beneficial. In addition, many Sirt1 deficient mice die 
from cardiac defects, which strengthens a potent role of Sirt1 with cardiac function.

Various pharmacological agents which includes resverterol to statins therapy improves cardioprotection in rodent models of myocardial infarction, suggest angiogenic and anti-apoptotic property of Sirt1, with anti-hyperlipidaemic role of the statins [26].

Although mitochondrial sirtuins such as Sirt4 and its role in electron transport have not been studied extensively recent reports illustrates importance of Sirt4. It is found in mitochondria and has no identified substrate but shows ADPribosyltransferase activity. Sirt4 binds adenine nucleotide translocator (ANT), which transports ATP into the cytosol and ADP into mitochondrial matrix, thereby providing a substrate for ATP synthase [35]. Yang et al. have reported that in cellular response to DNA damage when mitochondrial NAD+ level falls below critical level, Sirt4 displays anti-apoptotic activity protecting the cells from death. Thus significant increase in mitochondrial Sirt4 gene by ASA in our studies can inhibit all the pathways that consumes ATP and decrease apoptotic activity during cardiomyopathy. Furthermore, it has been reported that there is change in mitochondrial mass during heart disease, which suggest that mitochondrial biogenesis might protect diseased heart [36,37]. Thus an specific overexpression of PGC1 $\alpha$ and Tfam gene in HL1 cardiomyocyte by ASA treatment in our studies can prevent the process of mitochondrial polymorphism that develops during pathological state of organ.

Winnik et al. reported in his studies that an increase in Sirt1 can interfere with crucial steps of endothelial activation and can induce anti-inflammatory action to interfere with $\mathrm{Nfkb}$ signaling pathway thus preventing the foam cell formation. They also suggested that the findings can serve protective role of Sirt1 in atherogenesis and thrombosis.

Sirt4 also functions upstream of Sirt1 and AMPK which can have beneficial role in energy metabolism [35]. Thus these findings suggest us that it may be beneficial in treatment of artherosclerosis, cardiomyopathy and myocardial infarction. Our findings delineate a new role of $\mathrm{H}_{2} \mathrm{O}_{2}$ production, which despite its potential toxicity, can serve important in signal transduction and can also participate in retrograde activation of mitochondrial biogenesis. Low levels of ROS generated by ASA treatment can be beneficial as it improves energetic demand during myocadial ischemia [29].

Furthermore it has also been speculated that at low concentration ( $\leq 0.3 \mathrm{mM}$ ) ASA increases $\mathrm{Ca}^{2+}$ influx and augments long-lasting voltage gated L-type $\mathrm{Ca}^{2+}$ channels (LTCCS) of excitable cells, however at higher concentration $(>1$ $\mathrm{mM}$ ) it suppressed $\mathrm{Ca}^{2+}$ influx and LTCC activity. In electrically non-excitable mast cells it is believesd that store-operated $\mathrm{Ca}^{+}$ entry (SOCE) is main mode of $\mathrm{Ca}^{2+}$ influx. At low concentration ASA can also reduce $\mathrm{Ca}^{2+}$ release-activated $\mathrm{Ca}^{2+}$ (CRAC) channel activity. Thus, ASA may stimulate another $\mathrm{Ca}^{2+}$ entry pathway $[38,39]$.

\section{Conclusion}

In present studies of aspirin treatment to HL1 cardiomyocytes the drug at given dose decreases $A h R$ gene expression that would increase anti-inflammatory action and provide substantial benefit to heart by increasing Sirt1, PGC1 $\alpha$ gene expression and GPx and Catalase antioxidant status. The treament also increases mitochondrail biogenesis that can improve cardiac energetic demand suggestive of ASA might act as a potent antiartherosclerotic agent.

\section{References}

1. Yu W, Fu YC, Chen CJ, Wang X, Wang W (2009) SIRT1: A novel target to prevent atherosclerosis. J Cell Biochem 108: 10-13.

2. Yusuf S, Reddy S, Ounpuu S, Anand S (2001) Global burden of cardiovascular diseases: part I: general considerations, the epidemiologic transition, risk factors and impact of urbanization. Circ Res 104: 2746-2753.

3. Fontana L, Meyer TE, Klein S, Holloszy JO (2009) Long-term calorie restriction is highly effective in reducing the risk for atherosclerosis in humans. Proc Natl Acad Sci 17: 6659-6663.

4. Piantadosi CA, Carraway MS, Babiker A, Suliman HB (2008) Heme Oxygenase-1 Regulates Cardiac Mitochondrial Biogenesis via Nrf2Mediated Transcriptional Control of Nuclear Respiratory Factor-1. Circ Res 103: 1232-1240.

5. Suliman HB, Carraway MS, Ali AS, Reynolds CM, Welty-Wolf KE (2007) The CO/HO system reverses inhibition of mitochondrial biogenesis and prevents murine doxorubicin cardiomyopathy. J Clin Invest 117: 3730-3741.

6. Suliman HB, Carraway MS, Tatro LG, Piantadosi CA (2007) A new activating role for $\mathrm{CO}$ in cardiac mitochondrial biogenesis. J Cell Sci 120: 299-308.

7. Liu H, Jiang D, Zhang S, Ou B (2010) Aspirin inhibits fractalkine expression in atherosclerotic plaques and reduces atherosclerosis in ApoE gene knockout mice. Cardiovasc Drugs Ther 24: 17-24.

8. Khan Q, Mehta JL (2005) Relevance of platelet-independent effects of aspirin to its salutary effect in atherosclerosis-related events. J Atheroscler Thromb 12: 185-190.

9. Grootveld M, Halliwell B (1986) Aromatic hydroxylation as a potential measure of hydroxyl-radical formation in vivo. Identification of hydroxylated derivatives of salicylate in human body fluids. Biochem J 237: 499-504.

10. Kamble P, Selvarajan K, Narasimhulu CA, Parthasarathy S (2013) Aspirin may promote mitochondrial biogenesis via the production of hydrogen peroxide and the induction of Sirtuin1/PGC-1 $\alpha$ genes. Eur J Pharmocal 699: 55-61.

11. Verdin E, Hirschey MD, Finley LW, Haigis MC (2010) Sirtuin regulation of mitochondria: energy production, apoptosis, and signaling. Trends Biochem Sci 35: 669-675.

12. Haigis M, Mostoslavsky R, Haigis KM, Fahie K, Christodoulou DC, et al. (2006) Sirt4 inhibits glutamate dehydrogenase and opposes the effects of calorie restriction in pancreatic cells. Cell 126: 941-954.

13. Lin J, Puigserver P, Donovan J, Tarr P, Spiegelman BM (2005) Peroxisome Proliferator-activated Receptor $\gamma$ Coactivator $1 \beta$ (PGC-1 $\beta), \quad$ A Novel PGC-1-related Transcription Coactivator Associated with Host Cell Factor. J Biol Chem 277: 1645-1648. 
14. Puigserver P, Wu Z, Park CW, Graves R, Wright M, et al. (1998) A cold-inducible coactivator of nuclear receptors linked to adaptive thermogenesis. Cell 92: 829-839.

15. Nemoto S, Fergusson MM, Finkel T (2005) Sirt1 functionally interacts with the metabolic regulator and transcriptional coactivator PGC- 1\{alpha\}. J Biol Chem 280: 16456-16460.

16. Grube, Meyer zu Schwabedissen HE, Präger D, Haney J, Möritz KU, et al. (2006) Uptake of Cardiovascular Drugs Into the Human Heart Expression, Regulation, and Function of the Carnitine Transporter OCTN2 (SLC22A5). Circ 113: 1114-1122

17. Alcendor RR, Kirshenbaum LA, Shin-ichiro I, Vatner SF, Sadoshima J (2004) Silent information regulator $2 \alpha$, a longevity factor and class III histone deacetylase, is an essential endogenous apoptosis inhibitor in cardiac myocytes. Circ Res 95: 971-980.

18. Kwon H, Ott M (2008) The ups and downs of SIRT1. Trends Biochem Sci 33: 517-525.

19. Thannickal VJ, Fanburg BL (2000) Reactive oxygen species in cell signalling. Am J Physiol Lung Cell Mol Physiol 279: 1005-1028.

20. Zhang QJ, Wang Z, Chen HZ, Zhou S, Zheng W, et al. (2008) Endothelium-specific overexpression of class III deacetylase SIRT1 decreases atherosclerosis in apolipoprotein E-deficient mice. Cardiovasc Res 80: 191-199.

21. Meilhac O, Zhou M, Santanam N, Parthasarathy S (2000) Lipid peroxides induce expression of Catalase in cultured vascular cells. Jour Lipid Res 41: 1205-1213.

22. Rohrer DK, Kobilka BK (1998) G protein coupled receptors: functional and mechanistic insights through altered gene expression. Physiol Rev 78: 35-52.

23. Patterson C, Ruef J, Madamanchi NR, Barry-Lane P, Hu Z, et al. (1999) Runge Stimulation of a Vascular Smooth Muscle Cell NAD(P)H Oxidase by Thrombin Evidence that p47phox may participate in forming this oxidase in vitro and in vivo. The Journal of Biological Chemistry 274: 19814-19822.

24. Yao Z, Tong J, Tan X, Li C, Shao Z, et al. (1999) Schumacker PT Role of reactive oxygen species in acetylcholine-induced preconditioning in cardiomyocytes. Am Jour Physiol Heart Circ Physiol 277: 2504-2509.

25. Brandes RP (2008) Activating SIRT1: a new strategy to prevent atherosclerosis? Cardiovasc Res 80: 163-164.

26. Lavu S, Boss O, Elliott PJ, Lambert PD (2008) Sirtuins novel therapeutic targets to treat age-associated diseases. Nature Reviews Drug Discovery 7: 841-853.
27. Lin J, Handschin C, Spiegelman BM (2005) Metabolic control through the PGC-1 family of transcription coactivators. Cell Metab 1: $361-370$.

28. St-Pierre J, Lin J, Krauss S, Tarr PT, Yang R, et al. (2003) Bioenergetic analysis of peroxisome proliferator-activated receptor gamma coactivators 1alpha and 1beta (PGC-1alpha and PGC-1beta) in muscle cells. J Biol Chem 278: 26597-26603.

29. Klawitter PF, Murray HN, Clanton TL, Angelos MG (2002) Reactive oxygen species generated during myocardial ischemia enable the energetic recovery during reperfusion. Am J Physiol Heart Circ Physiol 283: 1656-1661.

30. Arany Z, He H, Lin J, Hoyer K, Handchin C, et al. (2005) Transcriptional coactivator PGC-1 alpha controls the energy state and contractile function of cardiac muscle. Cell Metab 1: 259-271.

31. Shoag J, Arany Z (2010) Regulation of Hypoxia-inducible genes by PGC-1 $\alpha$. Arterioscler Thromb Vasc Biol 30: 662-666.

32. Pillarisetti S (2008) A Review of Sirt1 and Sirt1 modulators in cardiovascular and metabolic diseases. Recent patents on cardiovascular drug discovery 3: 156-164.

33. Li L, Zhao L, Yi-Ming W, Yu YS, Xia CY, et al. (2009) Sirt1 hyperexpression in SHR heart related to left ventricular hypertrophy. Can J Physiol Pharmacol 87: 56-62.

34. Jaichander K, Selvarajan K, Garelnabi M, Parthasarathy S (2008) Induction of paraoxonase 1 and apolipoprotein $A 1$ gene expression by ASA. J Lipid Res 200: 1-26.

35. Verdin E, Hirschey MD, Finley LW, Haigis MC (2010) Sirtuin regulation of mitochondria: energy production, apoptosis, and signaling. Trends Biochem Sci 35: 669-675.

36. Yang H, Yang T, Baur JA, Perez E, Matsui T, et al. (2007) Nutrientsensitive mitochondrial NAD+ levels dictate cell survival. Cell 130: 1095-1107.

37. Davidson SM (2010) Endothelial mitochondria and heart disease. Cardiovasc Res 88: 58-66.

38. Winnik S, Stein S, Matter CM (2012) SIRT1-An anti-inflammatory pathway at the crossroads between metabolic disease and atheroscelerosis. Curr Vas Pharmacol 10: 693-696.

39. Suzuki Y, Inoue T, Ra C (2010) NSAIDs, Mitochondria and Calcium Signaling: Special Focus on Aspirin/Salicylates. Pharmaceuticals 3: 1594-1613. 